\title{
ERRATUM
}

Erol Uzal • L. Emir Sakman

\section{Erratum to: Dynamic response of a circular plate to a moving load}

Published online: 7 November 2009

(C) Springer-Verlag 2009

\section{Erratum to: Acta Mech}

DOI 10.1007/s00707-009-0207-y

Owing to an unfortunate oversight, one of the boundary conditions of this paper was omitted. Consequently, Eqs. (4), (7) and (15) contained errors. The correct equations are as follows:

$$
\begin{gathered}
\frac{\partial^{2} w}{\partial r^{2}}+\frac{v}{r} \frac{\partial w}{\partial r}=0, \\
\frac{\partial^{2} W}{\partial r^{2}}+\frac{v}{r} \frac{\partial W}{\partial r}=0, \\
I_{m}(\beta)\left[\frac{d^{2} J_{m}(\beta r)}{d r^{2}}+\frac{v}{r} \frac{d J_{m}(\beta r)}{d r}\right]-J_{m}(\beta)\left[\frac{d^{2} I_{m}(\beta r)}{d r^{2}}+\frac{v}{r} \frac{d I_{m}(\beta r)}{d r}\right]=0 .
\end{gathered}
$$

The online version of the original article can be found under doi:10.1007/s00707-009-0207-y.

E. Uzal $(\varangle) \cdot$ L. E. Sakman

Department of Mechanical Engineering, Istanbul University, Istanbul, Turkey

E-mail: euzal@istanbul.edu.tr

Tel.: +90-212-5931941 